\title{
Energy Efficient Target Tracking Using Sleep Scheduling Mechanism for Wireless Sensor Network
}

\author{
Sam Jenifer.P ${ }^{1}$, Vijayalakshmi.P ${ }^{2}$ \\ 'hindusthan college of engineering and technology coimbatore) \\ ${ }^{2}$ Assistant Professor hindusthan college of engineering and technology coimbatore)
}

\begin{abstract}
In the development of various large-scale Wireless Sensor Network systems, a particular challenging problem is how to dynamically organize the sensors network and route sensed information from the field sensors to a target system. A target tracking system is often required to ensure continuous monitoring, there always exist nodes that can detect the target along its trajectory (e.g., with low detection delay or high coverage level). Therefore, the most stringent criterion of target tracking is to track with zero detection delay or 100 percent coverage. Since nodes often run on batteries that are generally difficult to be recharged once deployed, energy efficiency is a critical feature of WSNs for the purpose of extending the network lifetime.The prime motivation of the work proposed to develop a energy efficient target tracking schemes. Toward this objective, the project uses a new energy-efficient dynamic optimization-based sleep scheduling and target prediction technique for large scale sensor networks. A probability-based prediction and optimization-based sleep scheduling protocol (PPOSS) is proposed to improve energy efficiency. A cluster-based scheme is exploited for optimization-based sleep scheduling. At every sampling instant, only one cluster of sensors that located in the proximity of the target is activated, whereas the other sensors are inactive. To activate the most appropriate cluster a non myopic rule is used based on not only the target state prediction but also its future tendency. Finally, the effectiveness of the proposed approach is evaluated and compared with the state-of-the-art protocols in terms of tracking accuracy, inter-node communication, and computation complexity.
\end{abstract}

\section{WIRELESS SENSOR NETWORK}

\section{Introduction}

A Wireless Sensor Network (WSN) consists of spatially distributed are deployed to monitor the sensing field and gather information from physical or environmental condition, such as temperature, sound, vibration, pressure, motion or pollutants and to co-operatively pass their data through the network to a main location.

Traditionally, two approaches can be adopted to accomplish the data collection task: Direct communication, and Multi-hop forwarding. In the sensor nodes upload data directly to the sink through one-hop wireless communication, which may result in long communication distances and degrade the energy efficiency of sensor nodes. On the other hand, with multi-hop forwarding, data are reported to the sink through multiple relays, and the communication distance is reduced. However, since nodes near the sink generally have a much heavier forwarding load, their energy may be depleted very fast, which degrades the network performance.

The applications of WSNs can be found in diverse areas such as military (e.g., battle field surveillance), environmental protection (e.g., habitat monitoring), healthcare (e.g., telemonitoring of human physiological data), and home automation. Sensor nodes in a WSN constitute a wireless ad-hoc network, with one or a few sink nodes as the collection point(s) and bridge(s) to the base station(s). Every node in the network may create data periodically, on demand of base stations, or triggered by events. At the same time, every node may forward data that it receives toward sink nodes, which are often multiple hops away. A typical multi-hop architecture of WSNs is shown Figure 1

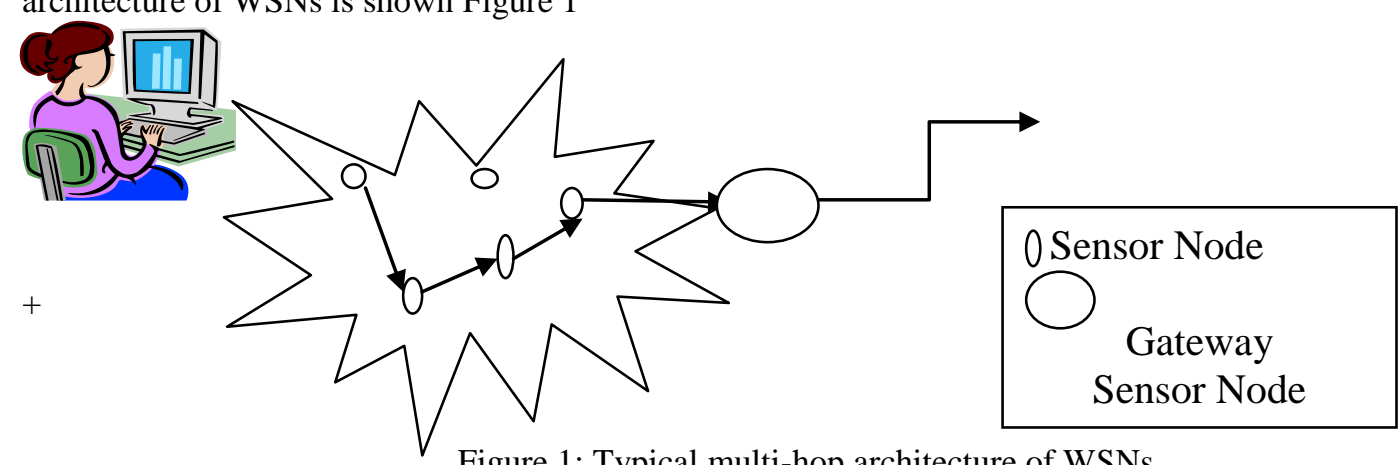

Figure 1: Typical multi-hop architecture of WSNs 


\section{Components of Sensor Networks}

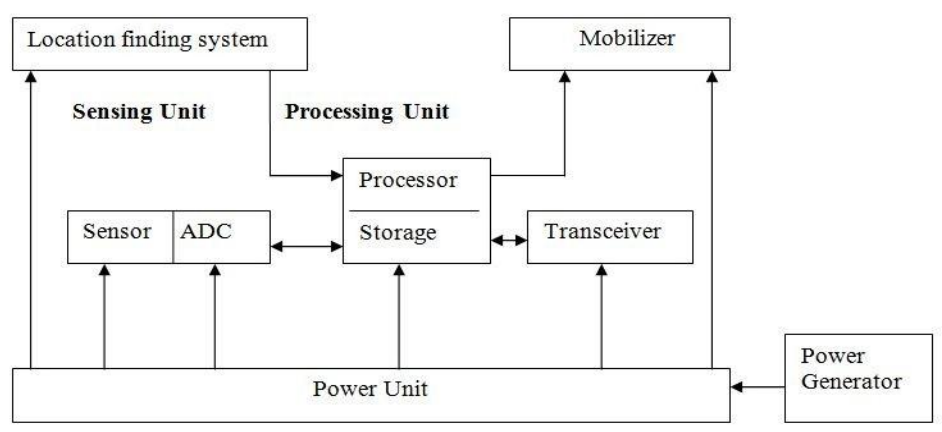

Fig.1.1.Components of sensor networks

Figure 2: Components of sensor networks

Sensing units are usually composed of two subunits: sensors and analog to digital converters (ADCs).The analog signals produced by the sensors are converted to digital signals by the ADC, and then fed into the processing unit.

\section{Arrangement of nodes in WSNs}

Sensor nodes are arranged in an ad-hoc manner in all the cases and the nodes have to arrange themselves in some spatial co-ordinate system. The nodes in the sensor networks are unaware of their location because they are arranged in an unplanned manner. Routing protocols can operate more efficiently by exploiting the location information. Global positioning system (GPS) is one of the solutions for this problem.

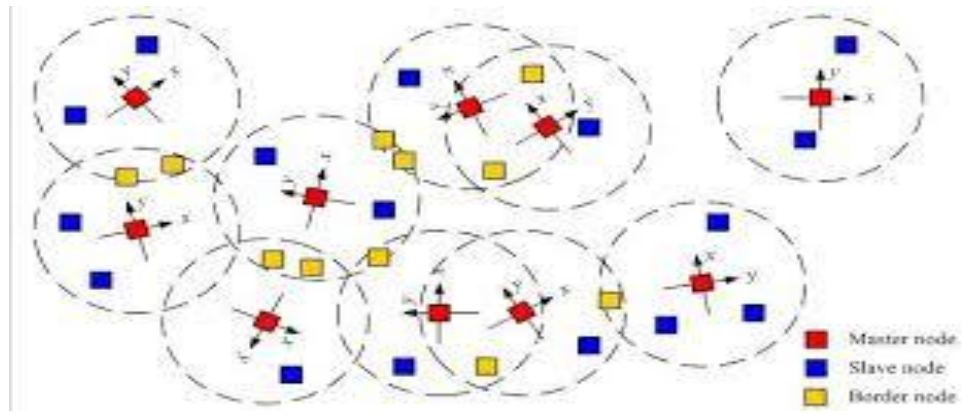

Figure 3: Arrangement of nodes in WSN's

- GPS can work only in outdoors.

- GPS receivers are expensive hence they are not appropriate in the construction of small cheap sensor nodes.

- In the presence of any obstacle like dense foliage etc, GPS cannot work.

Hence the sensor nodes have to organize themselves and establish their positions into a coordinate system without depending on an existing infrastructure. Localization is a problem has been studied for many years and it is an unavoidable problem when dealing with the sensor nodes. Depending upon the quality of localization, tracking attains fine-grained or coarse-grained location of the specific target.

\section{Area Monitoring}

Area monitoring is a common application of WSNs. In area monitoring, the WSN is deployed over a region where some phenomenon is to be monitored. A military example is the use of sensor to detect enemy intrusion; a civilian example is the geo-fencing of gas or oil pipelines. Similarly, wireless sensor network can use a range of sensors to detect the presence of vehicles ranging from motorcycles to train cars.

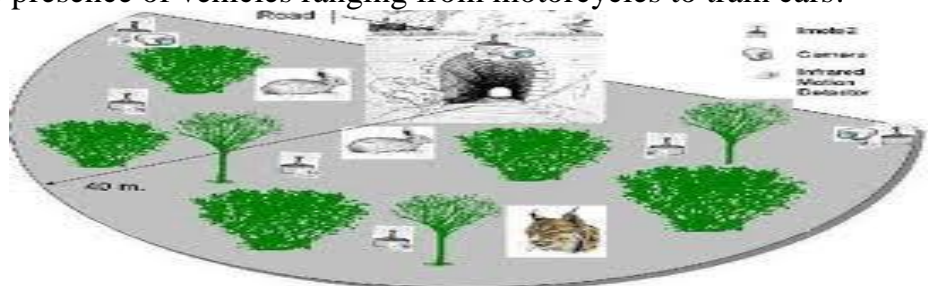

Figure 4: Area Monitoring 


\section{Environmental Sensing}

The term Environmental sensor network has evolved to cover many applications of WSNs to earth science research. This includes sensing volcanoes, oceans, glaciers, forests, etc.

\section{Forest Fire Detection}

A network of sensor nodes can be installed in a forest to detect when a fire has started. The nodes can be equipped with sensors to measure temperature, humidity and gases. Which are produced by fire in the trees or vegetation.The wireless sensor network, fire brigade will be able to know when a fire is started and how it is spreading.

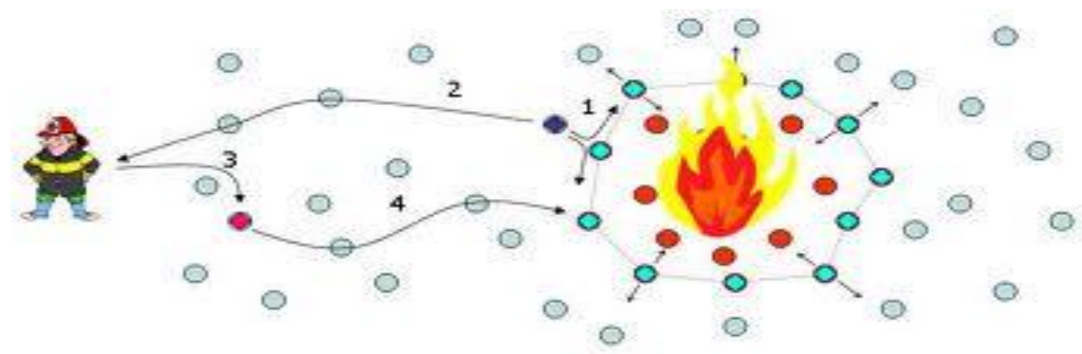

Figure 5: Forest Fire Detection

\section{Greenhouse Monitoring}

Wireless sensor network are also used to control the temperature and humidity levels inside commercial greenhouses. When the temperature and humidity drops below specific levels, the greenhouse manager must be notified Via e-Mail or cell phone text message, or host system can trigger misting systems, open vents, turn on fans or control a wide variety of system responses.

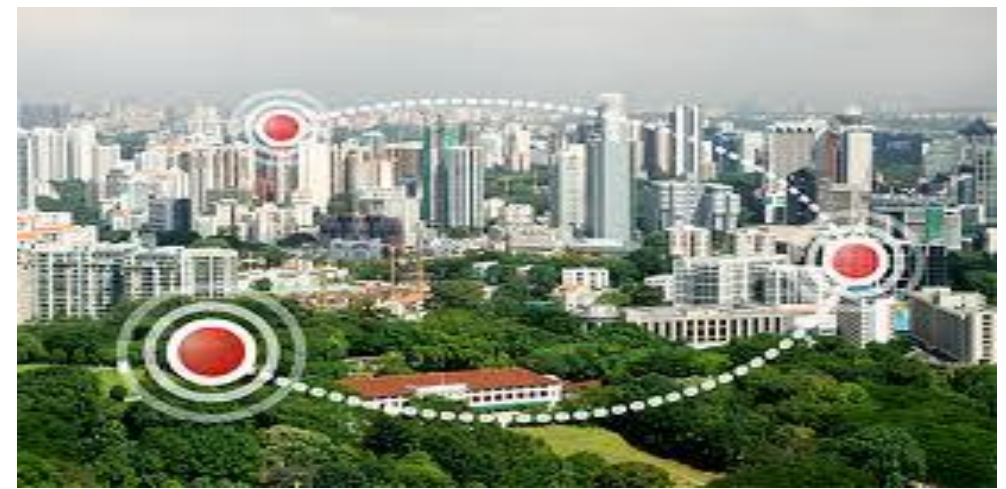

Figure 6: Greenhouse Monitoring

\section{II.Background Information}

This chapter mainly reviews the background information on energy efficiency, target tracking using probability prediction, and sleep scheduling.

\section{Target Tracking}

As one of the most important applications of WSNs, target tracking was widely studied from many perspectives. First, tracking was studied as a series of continuous localization operations in many existing efforts. Secondly, target tracking was sometimes considered as a dynamic state estimation problem on the trajectory, and Bayesian estimation methods, e.g., particle filtering, were used to obtain optimal or approximately optimal solutions.

Thirdly, in some cases, target tracking was considered as an objective application when corresponding performance metrics, e.g., energy efficiency or real-time feature, were the focus. Fourthly, a few efforts were conducted based on real implementation, and emphasized the actual measurement for a tracking application. Finally, a few target tracking efforts did not explicitly distinguish tracking from similar efforts, such as detection and classification. 


\section{Target Prediction}

Typical techniques for target prediction include kinematics-based prediction, dynamics-based prediction and Bayesian estimation methods.

Kinematics and dynamics are two branches of the classical mechanics. Kinematics describes the motion of objects without considering the circumstances that cause the motion, while dynamics studies the relationship between the object motion and its causes. In fact, most of past work about target prediction uses kinematics rules as the foundation, even for those that use Bayesian estimation methods.

\section{Energy Efficiency}

Since energy efficiency is a critical feature of WSNs, it has been extensively studied either independently or jointly with other features. Besides these efforts, energy efficiency is also considered as a constraint when designing algorithms or protocols for WSNs.

In the authors proposed, analyzed and evaluated the energy consumption models in WSNs with probabilistic distance distributions to optimize grid size and minimize energy consumption accurately. The models were also used to study variable-size grids, which can further improve the energy efficiency by balancing the relayed traffic in wireless sensor networks

\section{Sleep Scheduling}

In the past, there were many existing research efforts about sleep scheduling to prolong the network lifetime of WSNs. But since the first dissertation problem focuses on sleep scheduling for energy efficient target tracking, we mainly review those efforts that integrate sleep scheduling and target prediction.

\section{Existing System}

In spite of the diverse applications, WSNs face a number of unique technical challenges due to their inherent energy and bandwidth limitations, ad hoc deployment, and unattended operation, etc.,. Unfortunately, very little previous works on distributed systems can be applied to WSNs, since the underlying assumptions have changed dramatically. Therefore, innovative energy-aware, scalable, and robust algorithms for distributed signal processing in WSNs are highly required. A problem that is closely related is the localized topology control, which maintains energy-efficient network connectivity by controlling the transmission power at each node, or selecting a small subset of the local links of a node.

\section{Performance Evaluation \\ Performance Metrics}

Before reporting our evaluation results, we first define the metrics used to estimate energy efficiency and tracking performance. In the experiments, all of these metrics were examined for each target intrusion case.

1) Energy efficiency: Since the energy consumption of sleep scheduling is highly relative to the position where the target is detected, the energy consumptions on different nodes may vary significantly. Given this inequity, the network lifetime - time until the first sensor node runs out of power will be a less useful metric. Instead, we use the network-wide extra energy (EE) as the criterion of energy efficiency for sleep scheduling, which is defined as:

$$
E E=\sum_{i} E E i=\sum_{i}(\text { Escheduled }- \text { Edefault })
$$

Where $E_{\text {default }}$ is the energy consumption of node $i$ for idle listening when no target is detected, and $E_{\text {scheduled }}$ is the total energy of node $i$ consumed for sleep scheduling when a target is detected (e.g., for proactive wake-up, prolonged active time etc.). Both of them are measured during the same tracking period. As we focus on keeping tracking a target instead of data collection, neither of them involves the communication energy for propagating target information towards sink nodes.

2) Tracking performance (for the simulation): The tracking delay is one of the most important performance metrics for tracking. Since tracking is a process of continuous detections, the tracking delay with average detection delay $(\mathrm{AD})$, which is defined as the trajectory-wide average of escape times:

$$
A D=E[\Delta T]
$$

Where $\Delta T$ is the interval between the time when the target enters the surveillance field or gets lost, and the time when it is detected for the next time. Before the target is detected for the first time, PPSS protocol is not started and all the nodes work in the default duty cycling mode. Thus, the initial detection delay is out of the scope of PPSS's performance. We measure AD only after the first detection.

3) Tracking performance (for the implementation): In the prototype implementation, it is usually difficult to achieve precise time synchronization. Although need to implement time synchronization to run PPSS, the 
detection delay measurement is probably not precise enough to evaluate the tracking performance. Instead, of use escape distance percentage(EDP) as an

Alternative of $\mathrm{AD}$, which is defined as the percentage of a target's escape distance in the total trajectory:

$$
E D P=\sum D_{\text {escape }} / D_{\text {total }}
$$

Where $D_{\text {escape }}$ is the distance that none of the node scan detects the target, and $D_{\text {total }}$ is the total length of the target's trajectory. Thus, a low escape distance Percentage will mean a good coverage on the target's trajectory.

\section{Simulation and Analysis}

This simulation evaluated the influence of the following factors on EE and AD of three protocols: node density $\rho$, target speed $v, \frac{R}{r}$ ratio, localization error $\mathcal{E}$, and target movement model.

1. Node density is the comparison of three protocols at various node densities. As the node density increases, EE increases, and $\mathrm{AD}$ decreases, because an increasing number of nodes are proactively awakened to track the target.

2. Target speed the evaluation results at various target speeds. The reason that EE decreases is that a faster target will stay shorter in the alarm node's communication range. AD will also decrease, because when the target moves faster, it will traverse the sensing range of more sensor nodes in the same period of time.

3. $\frac{R}{r}$ Ratio the simulation, we fixed $\mathrm{R}$ and decreased $\mathrm{r}$ to increase the $\frac{R}{r}$ ratio. When $\mathrm{r}$ decreases, the active time of awakened nodes will decrease according. Thus, the energy consumption will decrease too. At the same time, a decreasing $r$ will decrement the probability of detecting a target, therefore increment the detection delay.

4. Localization error show the evaluation results at various localization errors. We observe that the impact of the localization error on Circle scheme and MCTA is slight, because they awaken all the nodes in an area or a contour.

\section{Proposed System}

Our proposed work, present a probability-based target prediction and sleep scheduling protocol (PPSS) to improve the efficiency of proactive wake up and enhance the energy efficiency with limited loss on the tracking performance. With a target prediction scheme based on both kinematics rules and theory of probability, PPSS not only predicts a target's next location, but also describes the probabilities with which it moves along all the directions.

\section{Ppss Routing Protocol}

PPSS is designed based on proactive wake up: when a node (i.e., alarm node) detects a target, it broadcasts an alarm message to proactively awaken its neighbour nodes (i.e., awakened node) to prepare for the approaching target. To Enhance energy efficiency; we modify this basic proactive wake-up method to sleep-schedule nodes precisely. Specifically, PPSS selects some of the neighbour nodes (i.e., candidate node) that are likely to detect the target to awaken.. Reduce the number of awakened nodes.

2. Schedule their sleep pattern to shorten the active time.

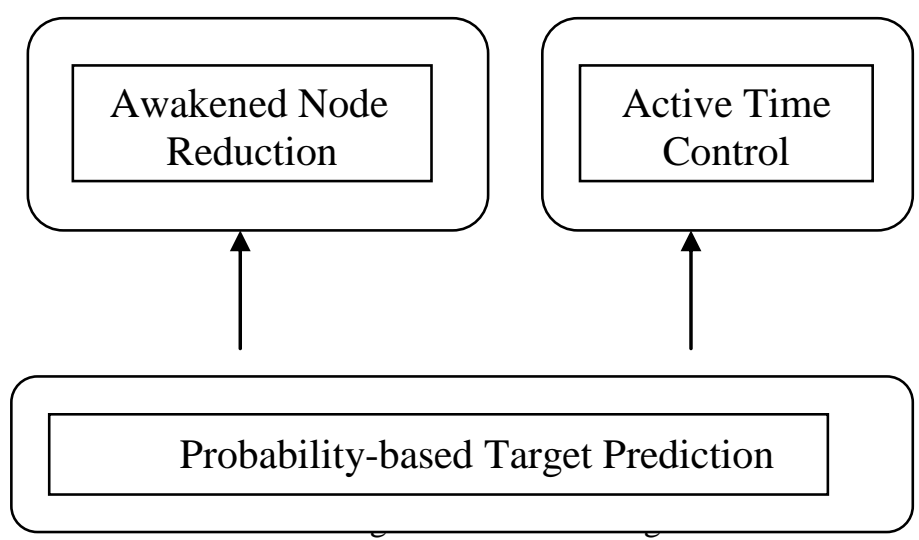

First, the number of awakened nodes can be reduced significantly, because: 1) Those nodes that the target may have already passed during the sleep delay do not need to be awakened; 2) Nodes that lie on a direction that the target has a low probability of passing by could be chosen to be awakened with a low probability. For this purpose, we introduce a concept of awaken region and a mechanism for computing the scope of an awake region. Second, the active time of chosen awakened nodes can be curtailed as much as possible, because they 
could wake up and keep active only when the target is expected to traverse their sensing area. For this purpose, we present a sleep scheduling protocol, which schedules the sleep patterns of awakened nodes individually according to their distance and direction away from the current motion state of the target.

1. Target prediction. The proposed target prediction scheme consists of three steps: current state calculation, kinematics-based prediction, and probability based prediction. After calculating the current state, the kinematics-based prediction step calculates the expected displacement from the current location within the next sleep delay, and the probability-base prediction step establishes probabilistic models for the scalar displacement and the deviation.

2. Awakened node reduction. The number of awakened nodes is reduced with two efforts: controlling the scope of awake regions, and choose a subset of nodes in an awake region.

3. Active time control. Based on the probabilistic models that are established with target prediction, PPSS schedules an awakened node to be active.

\section{Target Prediction}

1. Current state calculation: Based on State (n-1) and the current position that is assumed to be obtained by sensing or by calculating, PPSS calculates the current speed, direction, and acceleration, and finally composes the current

2. Kinematics-based prediction: Based on kinematics rule, PPSS predicts and the displacement (i.e., the displacement vector during. We denote the predicted value of a variable as its name with a prime symbol, is the predicted.

3. Probability-based prediction: When the displacement is described with its polar coordinate, we establish probabilistic models for the scalar displacement (i.e., the polar radius) and the deviation (i.e., the polar angle).

\section{Awakened Node Reduction}

Beyond the effort that limits the awakened nodes within an awake region, the number of awakened nodes can be further reduced by choosing only some nodes in the awake region as awakened nodes. By choosing an awakened node based on a probability related to the moving directions, awakened nodes can be reduced significantly.

1. Constrain the Awake Region Scope

Let d denote the distance of an awakened node from the alarm node, we next determine an awake region's scope by deciding the value scope of d. Here, we make the same approximate assumption that the target's position is exactly the alarm node's position to simplify the computation.

2. Awakened Nodes Selection

As discussed previously, only some of the member nodes in an awake region need to be awakened. By taking into account the prediction results on moving directions, we can further reduce the number of awakened nodes in an awake region so as to save more energy than solely constraining the scope of an awake region.

\section{Active Time Control}

After reducing the number of awakened nodes, energy efficiency can be enhanced further by scheduling the sleep patterns of awakened nodes, as not all the awakened nodes need to keep active all the time.

We schedule the sleep patterns of awakened nodes by setting a start time and an end time of the active period. Out of this active period, awakened nodes do not have to keep active. Therefore, the time that an awakened node has to keep active could be reduced compared with the Circle scheme.

\section{PPSS Protocol Finite State Machine}

States.

In the protocol core module of PPSS, we designed an internal finite state machine consisting of four

1) Default mode/IN DC: active in the default duty cycling mode;

2) Default mode/NOT IN DC: sleeping in the default duty cycling mode;

3) Tracking mode/IN T START: the sleep pattern is scheduled to sleep until tstart; and

4) Tracking mode/IN T END: the sleep pattern is scheduled to keep active until tend. 


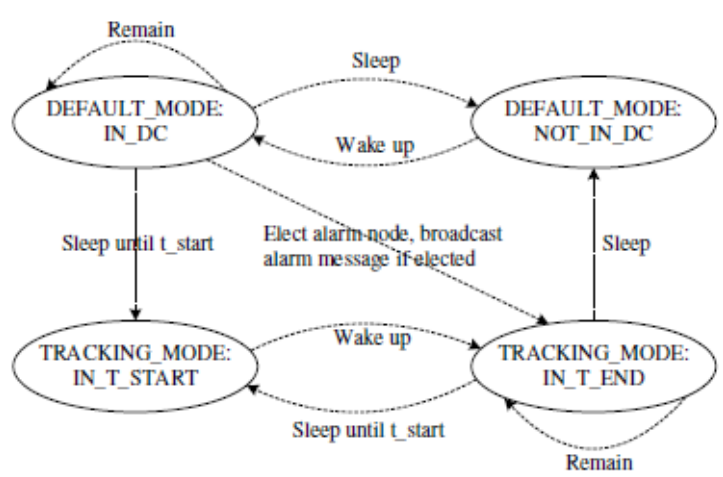

Figure 7 :PPSS protocol finite state machine

\section{Sensor Network Formation}

\section{V.Methodology}

We contribute to a more systematic understanding and treatment of sensor deployment issues. For this purpose, we studied the existing literature on deployment experience and present a classification of common problems encountered during deployment of sensor networks. A wireless network that is temporarily installed along a side the actual sensor network during the deployment process.

Parameters considered during sensor network formation

- Transmission range: nodes communication depends under transmission range which is placed nearly close to each other thus gets better link.

- Local information system: Nodes must be grouped under specific feature like battery power, processing capability, bandwidth, memory etc. so according to those, nodes are partitioned using driver methods.

- Mobility: Mobility refers the node movement procedure so need to consider the mobility options with limitation in maximum and minimum speed. According to the critical event monitoring process, sensor network formed under local information system with sleep wake scheduling mechanisms.

\section{Clustering and Approach Scheme}

A cluster-based scheme is proposed, where sensors are statically divided into clusters, and each cluster consists of a single Cluster Head $(\mathrm{CH})$ and a bunch of slave sensors. At every sampling instant, only one cluster of sensors is triggered to track the target. When a target enters the wireless sensor network, the $\mathrm{CH}$ that detects the target becomes active while other nodes are in sleep mode. Then the active $\mathrm{CH}$ selects three sensor nodes of its members for trackasing in which one node is selected as Leader node. The selected nodes sense the target and current target location is calculated.

\section{Routing Protocol Design}

A routing protocol is a protocol that specifies how routers communicate with each other, disseminating information that enables them to select routes between any two nodes on a computer network, the choice of the route being done by routing algorithms. Each router has a prior knowledge only of networks attached to it directly. A routing protocol shares this information first among immediate neighbors, and then throughout the network. This way, routers gain knowledge of the topology of the network. Design a Routing protocol named as PPOSS (Probability-based Prediction and Optimization-based Sleep Scheduling protocol), which is going to implement in OSI layer that need to get and deliver the messages from other layers for that make some more changes in supported layers. The routing protocol is implemented in the layered architecture of the NS2 simulator.

\section{Sleep Scheduling}

Measurements have shown that the energy that a sensor node spends while idly listening amounts to $50 \%-100 \%$ of the energy required for receiving. Furthermore, typically, a sensor node would spend a substantial fraction of the time in the idle state. Therefore, idle listening has been recognized as one of major sources of energy waste in sensor networks and sleep scheduling has been widely studied. The mainstream of research on sleep scheduling can be divided into two approaches. 


\section{Target Tracking}

This module quantifies the benefits of our approach in terms of energy consumed and accuracy of tracking for different mobility patterns. The key issues in tracking a mobile target are accuracy of tracking and energy expenditure. The accuracy of tracking is strongly influenced by the number of active sensor nodes. The more sensor nodes that are active, the higher will be the accuracy in tracking. Too few will result in inaccurate tracking. On the other hand, energy expenditure is proportional to the number of active sensor nodes; the larger the size of the active tracking region, the higher the energy consumption. To accurately track the target and minimize energy, a minimum set of sensors nodes need to be active

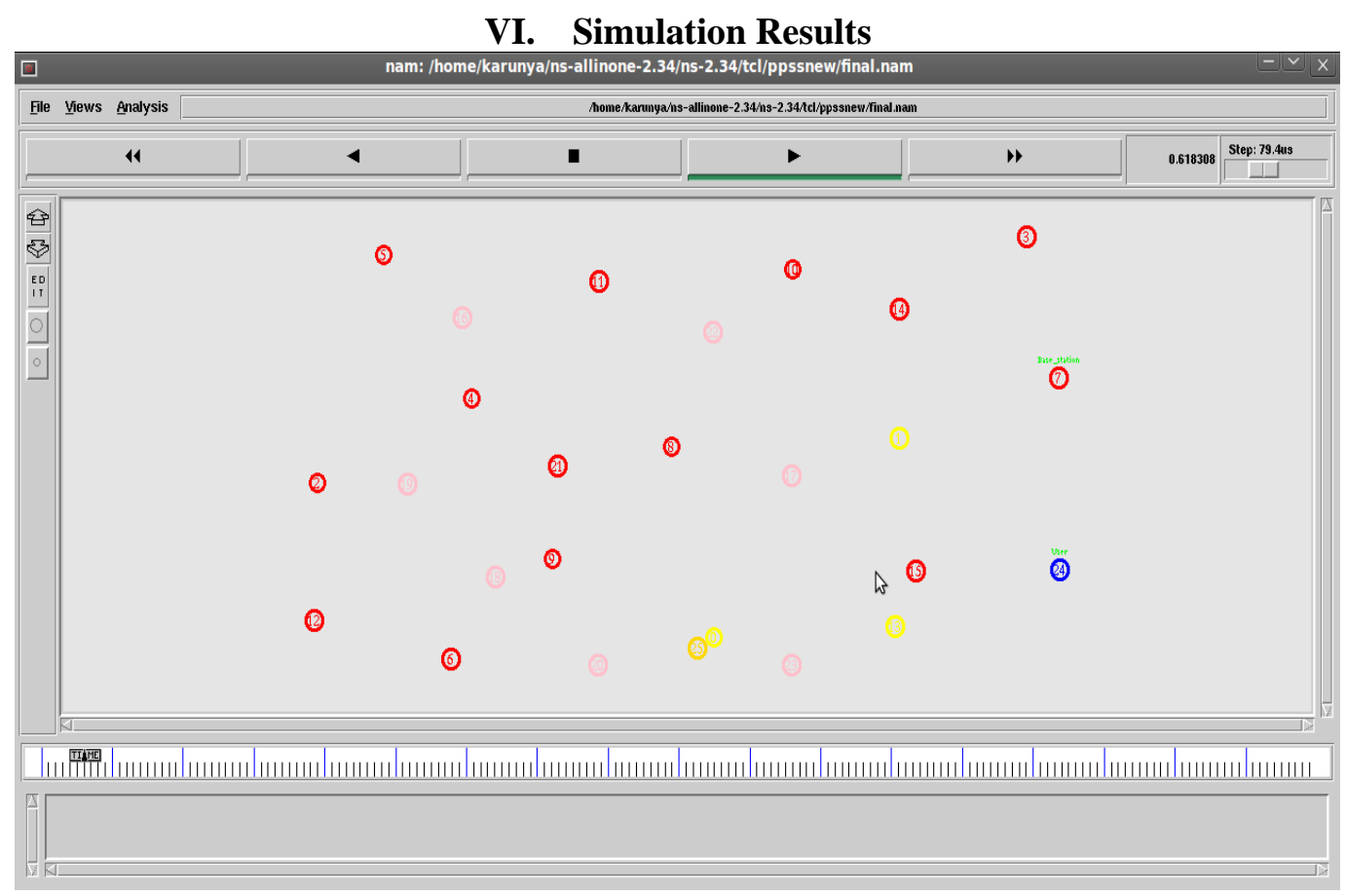

Figure 8: Simulation showing the basic data transmission set up

A single layer of awaken and sleep node is shown along with the nodes. Here nodes 7 base station, 24 user, and 25 object the sensing nodes.

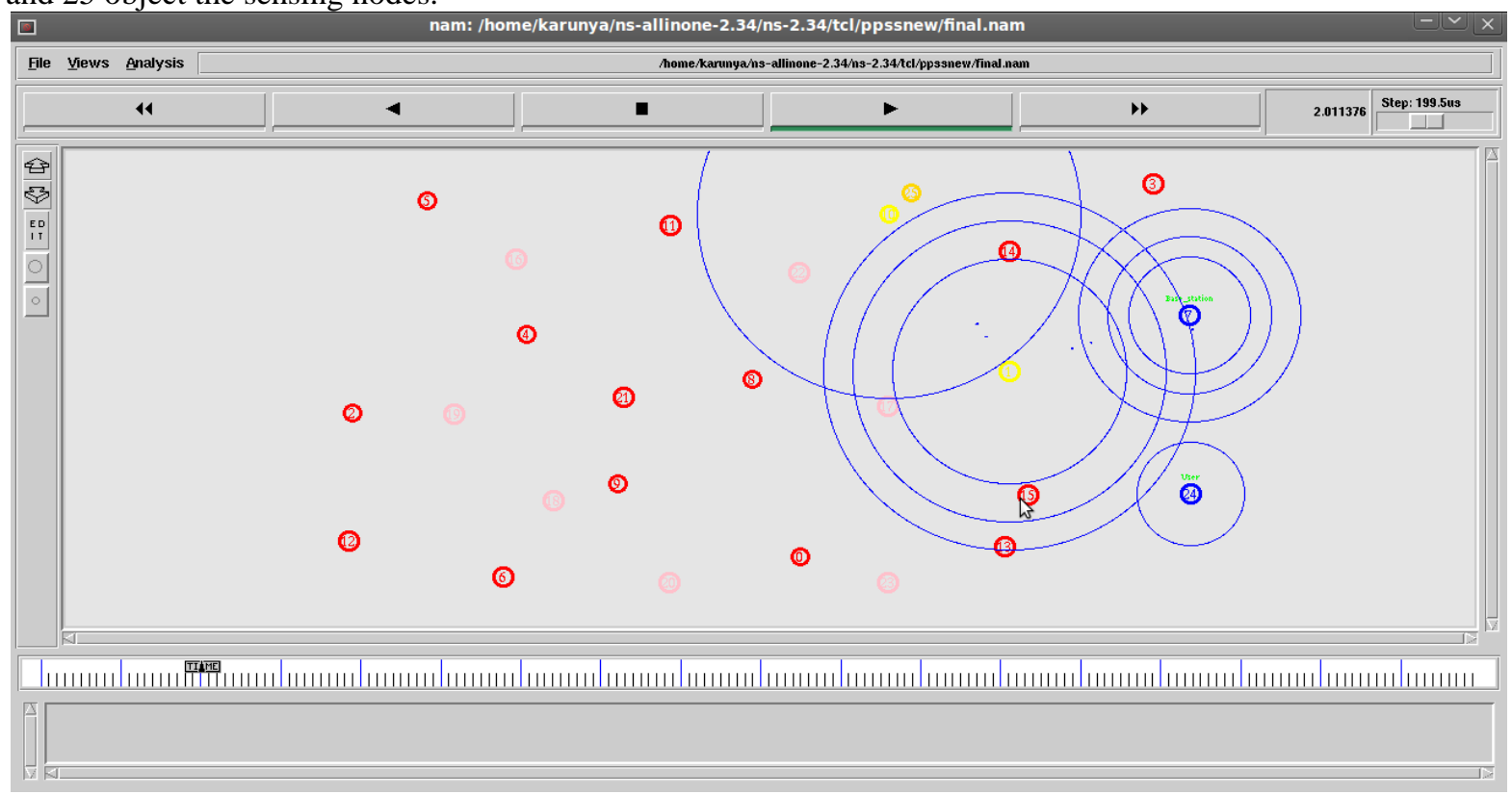

Figure 9: Simulation showing the transmission between the data.

Here there are total 25 nodes shown where nodes 15 and 16 of first layer are sending data to node 0 via the path $(15,16)-7-24$. 


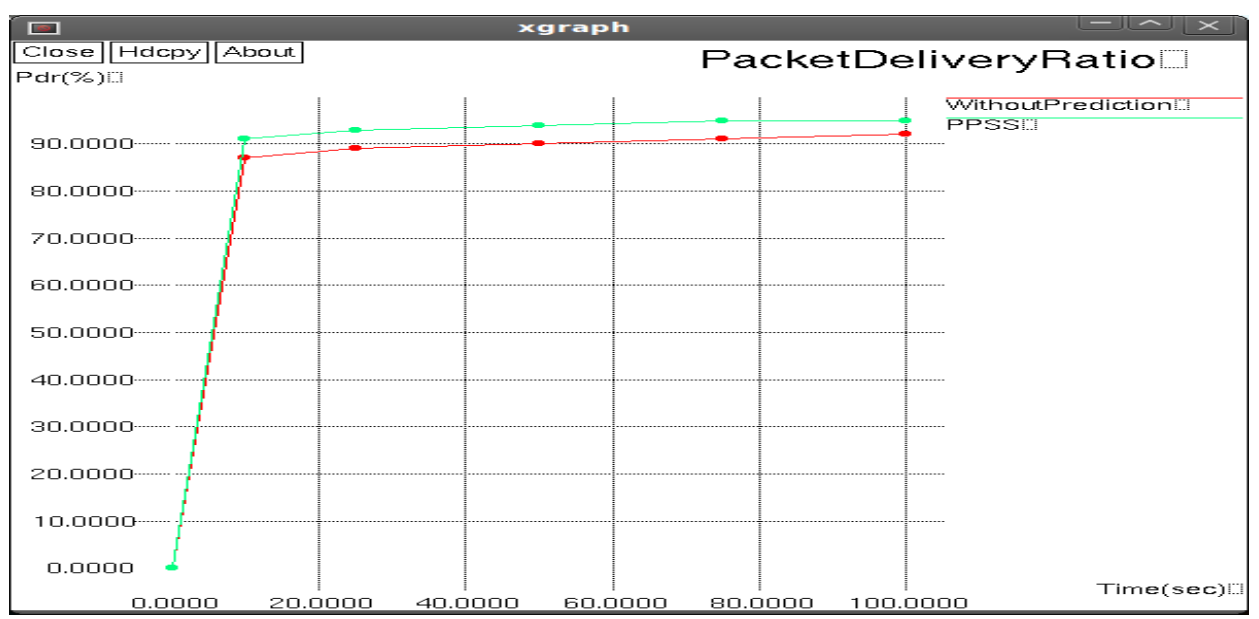

Figure 10: Simulation showing packet delivery ratio.

The existing without prediction scheme is compared with a PPSS protocol. It is found that packet delivery ratio is more for without prediction.

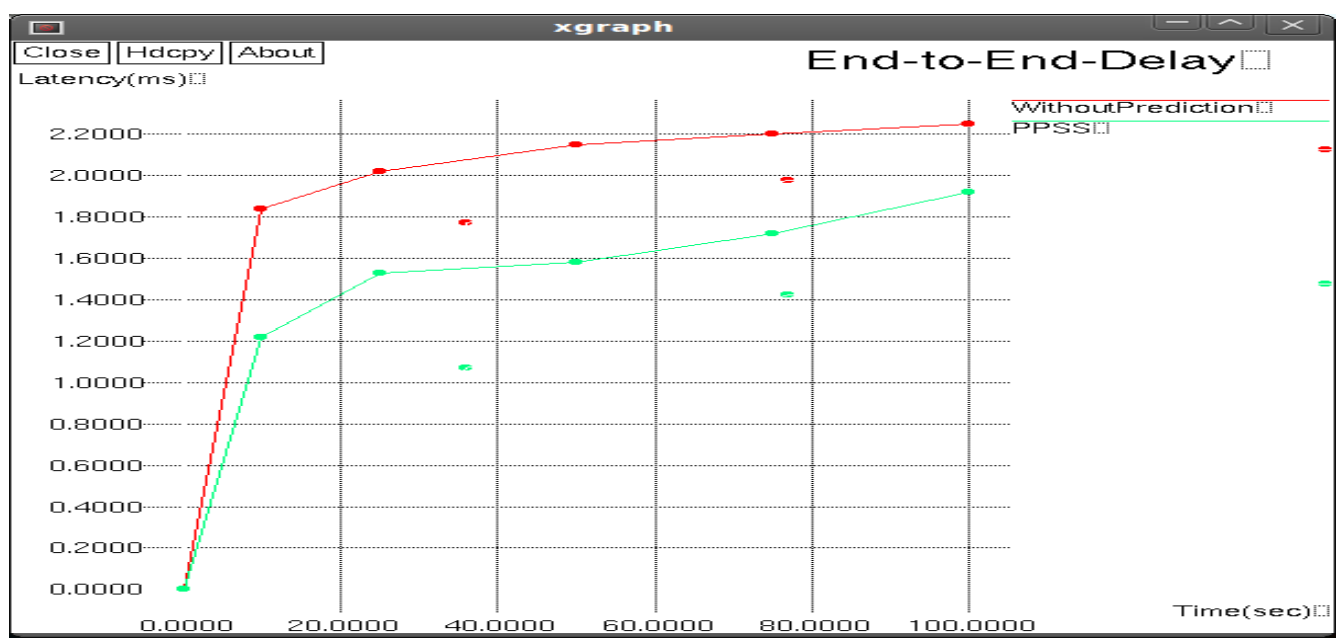

Figure 11: Simulation showing end to end delay.

Here the end to end delay for PPSS is lowered. As the reduce the number of awakened node beyond the threshold, it gets enabling efficient transmission with lesser delay compared to without prediction and PPSS layers.

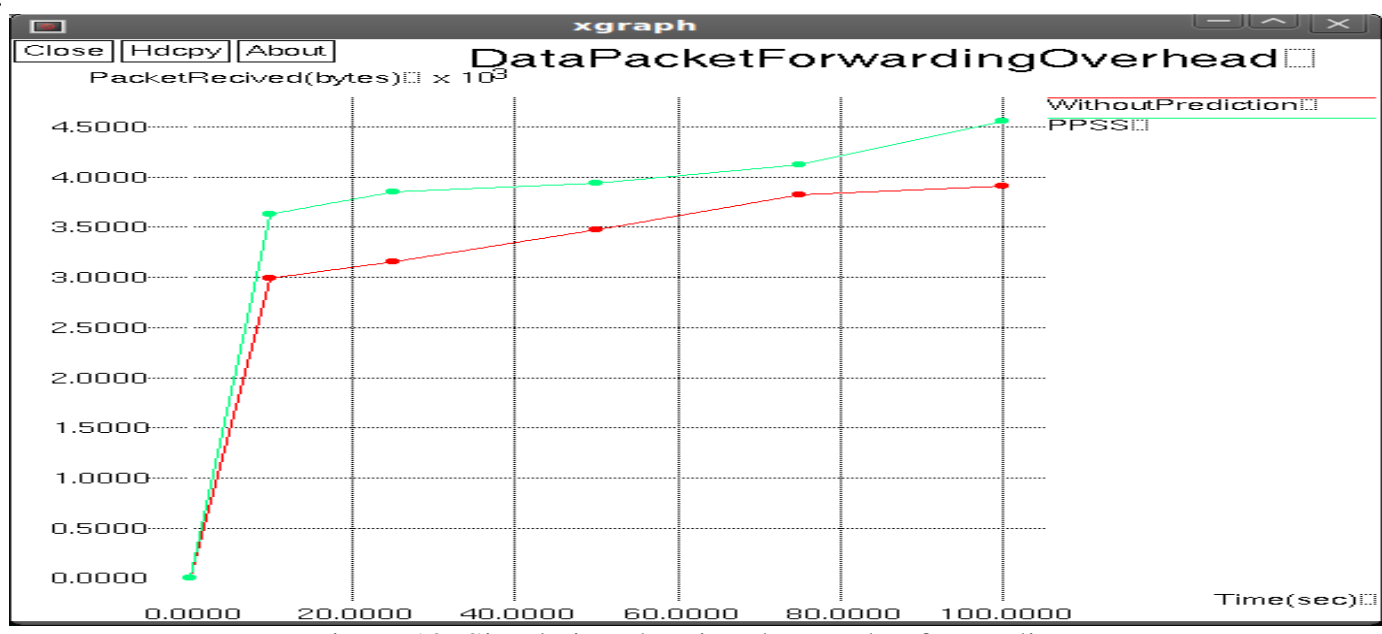

Figure 12: Simulation showing data packet forwarding.

The ratio of the number of received data packets to the number of total data packets sent by the source. 


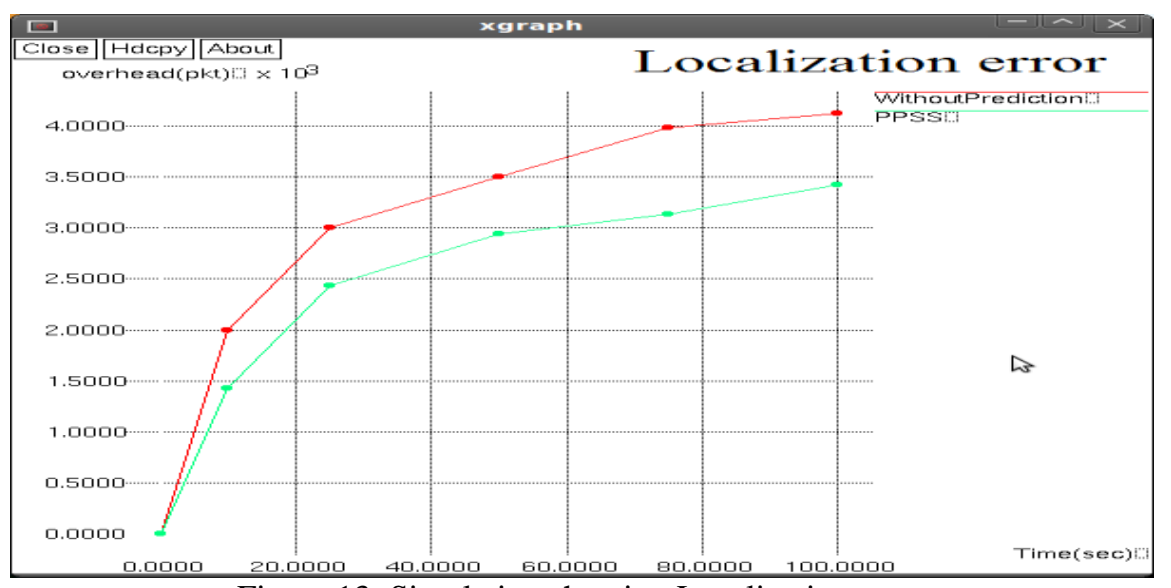

Figure 13: Simulation showing Localization error

The average number of transmitted control bytes per second, including both the data packet header and the control packets.

\section{VII.Conclusions And Future Enhancements}

In this project a system is developed in such a way that target tracking in WSN is done in efficient way using an energy efficient prediction based sleep scheduling algorithm. In a duty-cycled sensor network, proactive wake up and sleep scheduling can create a local active environment to provide guarantee for the tracking performance. By effectively limiting the scope of this local active environment (i.e., reducing low value-added nodes that have a low probability of detecting the target), PPSS improves the energy efficiency with an acceptable loss on the tracking performance. Given some limitations in tracking accuracy, the potential future work includes optimization-based sleep scheduling and target prediction for abrupt direction changes. So as a future enhancement, the tracking algorithm can be extended by forming clustering as one of the optimization methods.

\section{- PPSS has limitations as well.}

First, it does not use optimization methods, i.e., PPSS imposes no performance constraints when reducing the energy consumption. Without performance constraints, it is difficult to configure the protocol toward the best energy performance tradeoff for a specific network environment.

Second, the prediction method of PPSS cannot cover special cases such as the target movement with abrupt direction changes. This is the expense that PPSS pays for the energy efficiency enhancement. Given these limitations, the potential our work includes optimization-based sleep scheduling and target prediction.

In this approach three sensor nodes are selected each time in which two nodes calculates its distance from the moving object and sends the data to the leader node. The localization of the moving object is done by leader node whereas in previous methods it's done by $\mathrm{CH}$. Using prediction based clustering method energy consumed in the network will be reduced since the transmission power of the nodes is directly proportional to the distances. The three nodes selected for tracking are close to each other, thus the energy consumed for sending a data between the nodes is lower than sending a data from one of the selected nodes to its $\mathrm{CH}$.

In this work, a system is developed in such a way that target tracking in WSN is done in efficient way using an energy efficient prediction- based clustering algorithm. Energy efficient prediction based Clustering algorithm, reduces the average energy consumed by sensor nodes and thereby increase the lifetime of the network. The tracking of the moving object is accurately done.

\section{References}

[1] Akyildiz .I.F, Su .W, Sankarasubramaniam .Y, and Cayirci.E, (2002) "Wireless Sensor Networks: A Survey," Computer Networks, vol. 38, no. 4, pp. 393-422.

[2] Brownfield .M.I, Mehrjoo.K, Fayez .A.S, and Davis IV .N.J (2006) "Wireless Sensor Network Energy-Adaptive MAC Protocol"IEEE Communications Society subject matter experts for publication in the IEEE CCNC proceedings.

[3] Cao .Q, Yan .T, Stankovic .J, and Abdelzaher .T, (2005) "Analysis of Target Detection Performance for Wireless Sensor Networks,” Proc. Int'l Conf. Distributed Computing in Sensor Systems (DCOSS), pp. 276-292.

[4] Fuemmeler .J and Veeravalli.V, (2008) "Smart Sleeping Policies for Energy Efficient Tracking in Sensor Networks," IEEE Trans. Signal Processing, vol. 56, no. 5, pp. 2091-2101.

[5] Gui.C and Mohapatra.P, (2004) "Power Conservation and Quality of Surveillance in Target Tracking Sensor Networks," Proc. 10th Ann. Int'l Conf. Mobile Computing and Networking, pp. 129-143.

[6] Gu.Y and He.T, (2007) "Data Forwarding in Extremely Low Duty-Cycle Sensor Networks with Unreliable Communication Links," Proc. Fifth Int'l Conf. Embedded Networked Sensor Systems (SenSys ’07), pp. 321-334. 